\title{
Flexible Parylene-Based 3-D Coiled Cable
}

\author{
Ray Huang ${ }^{1}$, Student Member, IEEE and Yu-Chong Tai, Fellow, IEEE \\ Caltech Micromachining Laboratory, California Institute of Technology, USA
}

\begin{abstract}
Prosthesis systems require reliable and flexible connecting cables from the sensing/stimulating electrode sites to processing circuitries. However, the limitations on the fabrication materials and processes restrict the cables' ability to stretch, resulting in breakage and failure of the implanted cabled device. Thus, a microfabricated and fully implantable 3-D parylene coiled cable for prosthesis application is presented. Compared to traditional flexible cables, this parylene coiled structure is able to be stretched by $100 \%$ of its original length and is also long-term biocompatible. In addition, the cable structure can be heatformed in a mold to match muscle curvature and sharp turns in testing subjects and can also be directly integrated with flexible multi-electrodes arrays and neural probes.
\end{abstract}

Keywords: BioMEMS; flexible cable; parylene cable; neural prosthesis; interconnect

\section{INTRODUCTION}

Recent developments in neural prosthesis allow movement intention decoding by surgically implanting a sensing electrode array in the parietal cortex [1]. An insertion medium with flexible cable interconnect has been developed for this application [2], but along with several other state-of-the-art cable interconnect technologies [3-5], these wired interconnects are not able to accommodate the more physically active areas when implanted in testing subjects. Few researchers hypothesized that the extension, contraction and vibration of muscles groups often induce failures such as line fatigue, breakage and cable laceration during long term implantations. Thus, a biocompatible and stretchable alternative that is robust and reliable must be studied and developed.

In this work, we present the fabrication and testing results of a single metal layer parylene-based cable molded into a 3-D structure that is encapsulated with biocompatible silicone. Unlike previous versions of interconnects, this novel spiral structure provides ample room for the cable to be extended beyond its fabricated length and has the potential to overcome the aforementioned challenges. The fabrication process steps

This work was supported in part by National Science Foundation (award number H28927)

Contacting Author: Ray Huang is with the Caltech Micromachining Lab, California Institute of Technology, 1200 E. California Blvd, MC 136-93, Pasadena, CA 91125, USA. (Email: rhuang@ caltech.edu; phone:+1-626-3952227; fax:+1-626-584-9104) described in this work are fully compatible with MEMS technology and are able to be integrated with other system components. We have chosen to use parylene-C (poly-paraxylylene C) due to its flexibility and mechanical strength (Young's modulus $4 \mathrm{GPa}$, in between those of silicone and polyimide), insulation capability, its proven United States Pharmacopeia (USP) Class VI biocompatibility and its prior use in medical applications. In addition, parylene can be patterned using standard microfabrication techniques such as reactive ion etching.

\section{DEVICES DESIGN}

The fabricated parylene cables are $6 \mathrm{~cm}$ long and $0.9 \mathrm{~mm}$ wide. Each of these cables has 34 platinum trace lines that are 10 $\mu \mathrm{m}$ wide and $0.25 \mu \mathrm{m}$ thick with $10 \mu \mathrm{m}$ pitch embedded in two layers of parylene of equal thickness totaling $10 \mu \mathrm{m}$. The traditional cables provide little to no stress relief once implanted and fixed in the testing subject. In addition, the cables become more fragile after annealing treatment, which increase the chance of tearing and cracking which greatly reduces the life time of the cables. Our new proposed structure (Figure 1) consists of a heat-formed 3-D coil to alleviate these problems. After heat treatment the coils turns have diameters of $\sim 1.1 \mathrm{~mm}$ and pitches of $2 \mathrm{~mm}$. The number of turns and the diameters of the turns can be modified according to specification by changing the size of annealing rod. The cables are fabricated separately from the silicon probes and can be bonded together post-process (Figure 2). Both the silicon probe and the coiled cable structure provide metal bonding pads for electrical connection, which is satisfied by using commercially available conductive epoxy. Test cables with the above specifications are made in order to test the mechanical properties of this structure (Figure 3).

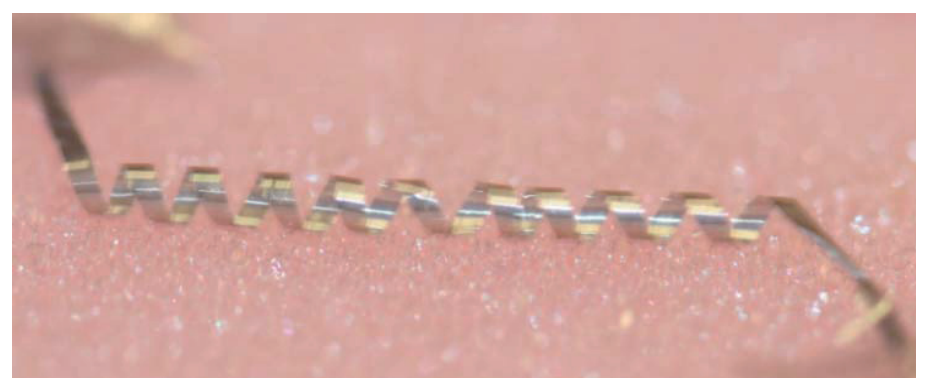

Figure 1. Close up view of the 3-D coiled parylene cable. The coil has 5 turns per centimeter and can be modified according to specification and surgery requirements. The cable can also be heat formed into other shapes and contours depending on the location of the implant. 


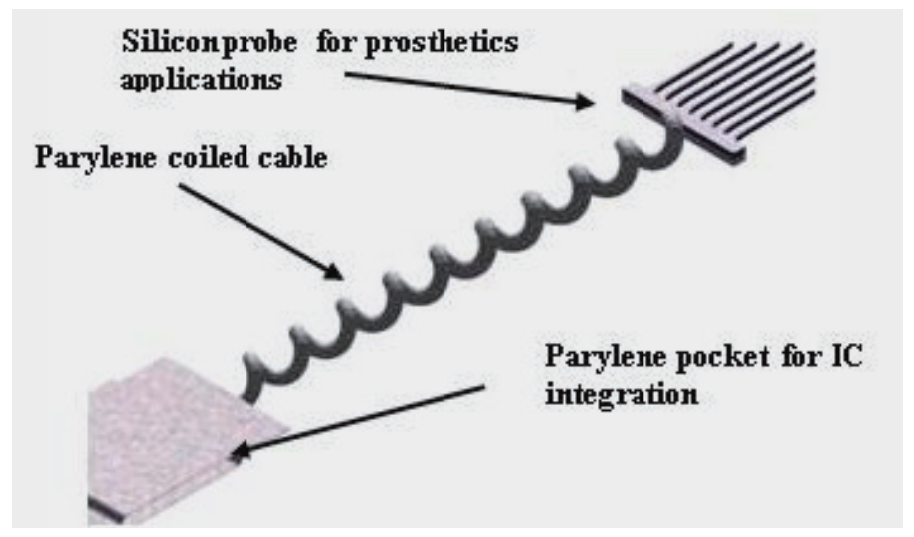

Figure 2. Concept schematic of the coiled parylene cable integrated with flexible parylene pocket for IC chip integration. The fabrication processes for the different components in the system are compatible and can be monolithically made. In this work, the silicon probe and the coiled cables are fabricated separately and are bonded together post-process.

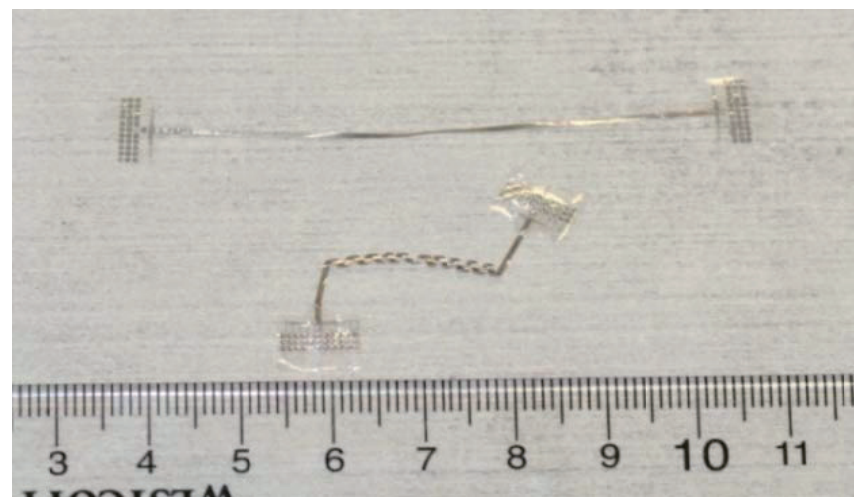

Figure 3. (top) Fabricated free standing parylene cable prior to heat treatment. (bottom) After heat treatment and 3-D molding. Its final fabricated length (cable only) is $2 \mathrm{~cm}$, compared to $6 \mathrm{~cm}$ before molding.

\section{FABRICATION}

The parylene cables are fabricated on single side polished wafers with RIE technology. Figure 4 shows the fabrication process [2]. A photoresist sacrificial layer is optionally spun on the wafer. Bottom layer parylene $\mathrm{C}$ of $\sim 5 \mu \mathrm{m}$ is vapor-deposited. Lift-off photoresist is subsequently spun on top of the parylene and exposed in a 10x reduction stepper under UV light. This lithography step is used to lift-off the electron-beam evaporated platinum $(0.25 \mu \mathrm{m})$, which defines the trace lines and connector pads. Top layer of parylene $C(5 \mu \mathrm{m})$ is deposited after the liftoff step to complete the parylene-metal-parylene sandwich structure. Photoresist is then spun and exposed to define the contact pads and the shape of the device. The exposed parylene is etched away by reactive ion etching (RIE) with $\mathrm{O}_{2}$ Plasma process. The wafer is finally soaked in a solvent to rid of the photoresist residue. The cables, upon releasing from the wafer, are individually hand-wrapped around a $1.1 \mathrm{~mm}$ diameter aluminum-foil-covered glass tube and heat treated for 48 hours at $200^{\circ} \mathrm{C}$ in vacuum with nitrogen backfill. The heat treatment also enhances the parylene to parylene adhesion.

After the heat treatment, the coiled cable is then dipped in a biocompatible silicone (Corning MDX4-4210) and cured in air and room temperature for two days for support and insulation (Figure 5). The two-part silicone is placed in a dish and mixed thoroughly with a stirring bar. The final mixture is then degassed in a bench-top vacuum chamber to rid of air bubbles to avoid potential failure points after coating.
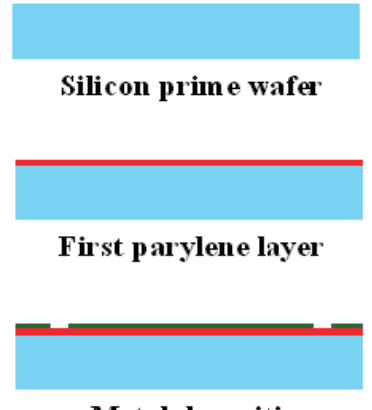

Metal dep osition

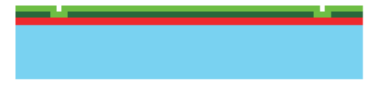

Second parylene layer

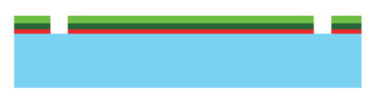

RIE op ening etch

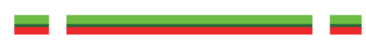

PR release

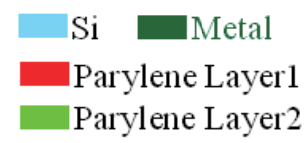

Figure 4. Parylene cable fabrication steps.

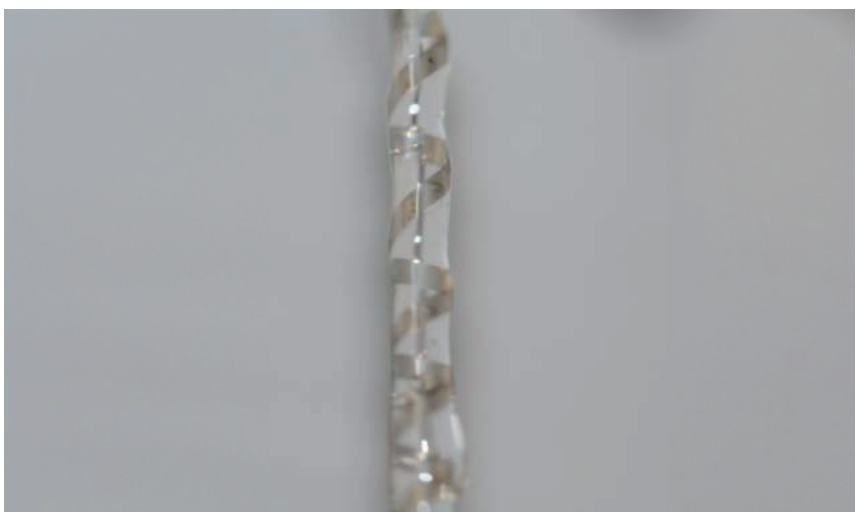

Figure 5. Silicone filled coiled parylene cable. The cable is dipped into silicone and pulled out to form the finished shape. The structure is then cured in air at room temperature for 48 hours.

\section{RESULTS AND DISCUSSION}

In order to measure and compare the effect of each treatment (coiling and silicone filling), several cable samples are made, which includes: regular 2-D cable, regular 2-D cable coated with silicon, heat-formed 3-D coil cable, and heat-formed 3-D coil cable filled with silicone. 
Mechanical and electrical tests are done on the samples. First, the samples are stretched along the axis of the cable and its maximum distance stretched before electrical failure (open circuit) is recorded (Figure 6). It is found that the traditional 2-D cables are not able to be stretched by more than $1 \%$ of its fabricated length before failure; regular cable coated with biocompatible silicone provided similar results. It is observed that the encapsulated platinum metal trace lines break before the parylene sandwich does. This is due to the difference in the tensile modulus (Parylene $\mathrm{C}$ has a Young's Modulus of $3.2 \mathrm{GPa}$, compare to Platinum's Young's Modulus of $168 \mathrm{GPa}$ ).

On the other hand, our coiled cables are able to be stretched by more than $100 \%$ of its fabricated length before the traces are broken (Figure 7). Even when the 3-D cables are filled with silicone, it is still able to extend by approximately $20 \%$ of its fabricated length. The yield strength along the axis of the cable is also measured. The result of the tests are summarizes in Table 1 .

One should note that by adjusting the diameter of the annealing rod, we can change the size of the loops of the coil, thus giving different spring constant and thickness for different prosthetic applications. A cross section of the final silicone filled cable is shown in Figure 8. The ratio of the length of the straight 2-D cable and that of the coiled cables is $3: 1$, which means for every unit length of 3-D coiled cable made, we need three times as much 2-D cable length. The cable is bonded to a siliconprobe-only structure (Figure 9) to demonstrate system integration. Conductive epoxy is used to connect the metal pads from the cable and the bonding pads on the silicon probe structure. The impedances of the electrodes are successfully measured via the coiled cable; sine wave signals passed into the saline solution are also recovered. The platinum electrodes [2] on the silicon probe shank have dimensions of $20 \mu \mathrm{m} \times 20 \mu \mathrm{m}$ and are fabricated in an array of four.

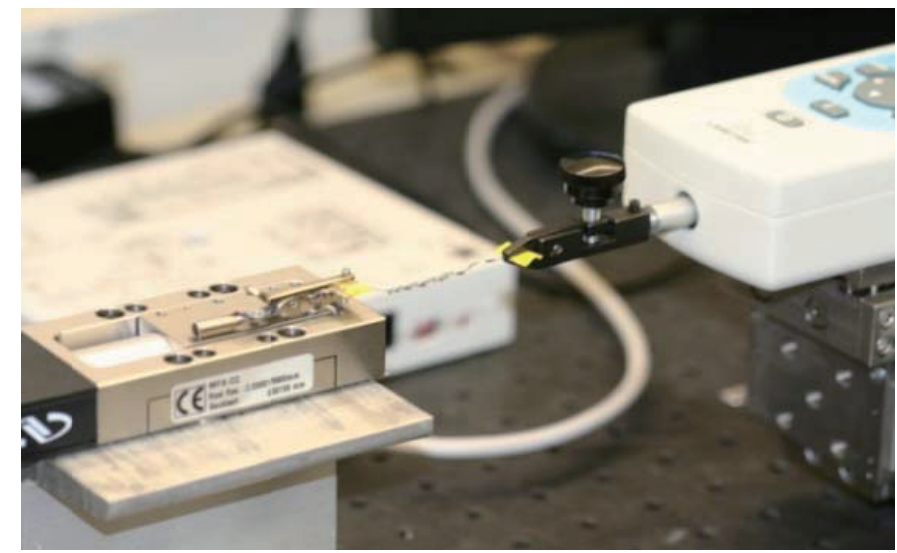

Figure 6. Mechanical test setup for coiled parylene cable. Electrical failure is determined by the value of the cable line resistance. A setup consists of an actuator and a force gauge is used to conduct this experiment.

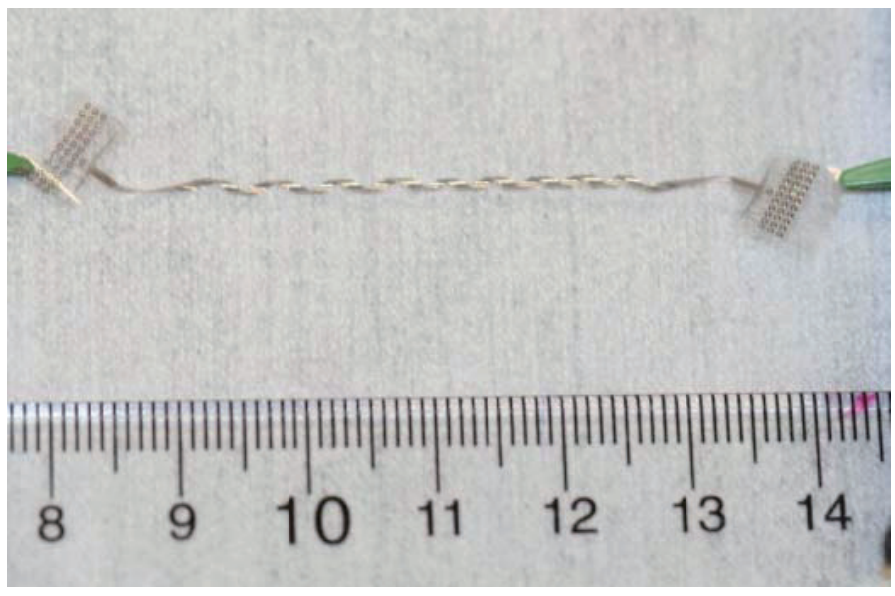

Figure 7. Cable after stretching. Compared to the relaxed state, the coiled cable is able to stretch for more than $100 \%$ of its original fabricated length

Finally, the mean time to failure of the cable is determined by soaking the cable in saline solution at various temperatures $\left(60^{\circ} \mathrm{C}, 80^{\circ} \mathrm{C}\right.$ and $\left.90^{\circ} \mathrm{C}\right)$. On-going passive testing results show that the 3-D coiled, silicone filled cable can survive more than 20 days in $90^{\circ} \mathrm{C}$ without any compromise in both electrical and mechanical properties. Figure 10 shows the electrical resistance of the platinum lines as a function of days soaked in saline solution. It is clear from the result that no noticeable resistance fluctuation occurs during this period; this result further validates the functionality and reliability of the fabricated structure.

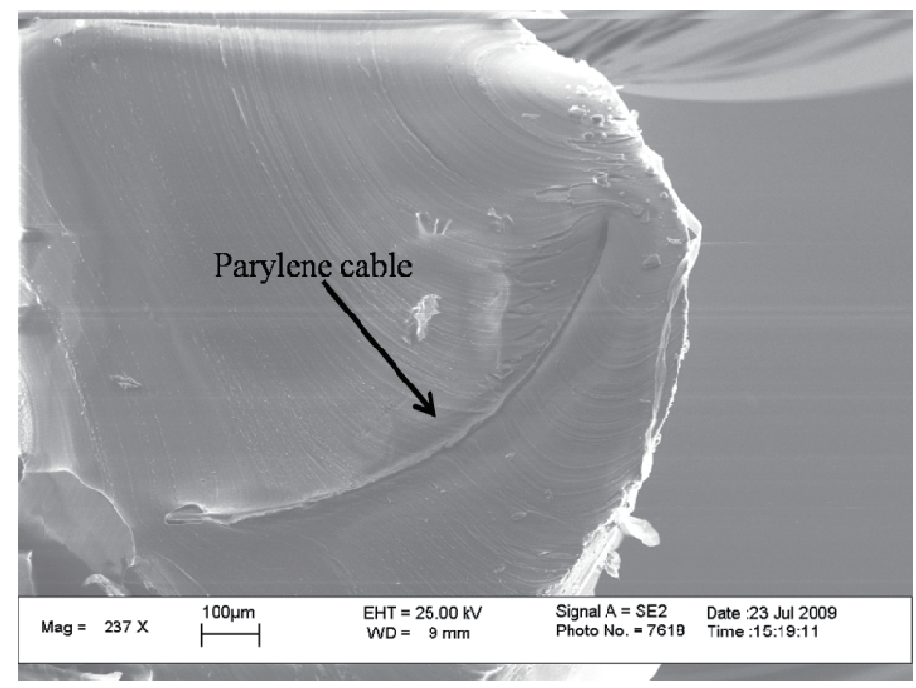

Figure 8. SEM picture of the cross section of silicone-enhanced coiled cable. The thickness of the cable is $10 \mu \mathrm{m}$. One should note that the cable is encapsulated inside the silicone in a spiral fashion, which results in an offcentered cable cross section as shown in the SEM. 
Table 1. Comparison of the traditional parylene cable vs. the new cable

\begin{tabular}{|c|c|c|c|}
\hline & Traditional Cable & Coiled Cable & Silicone Filled Coiled Cable \\
\hline Cross Section Dimension & $10 \mu \mathrm{m} \times 0.9 \mathrm{~mm}$ & $1.1 \mathrm{~mm}$ diameter & $1.4 \mathrm{~mm}$ diameter \\
\hline Fabricated Length & $6 \mathrm{~cm}$ & $2 \mathrm{~cm}$ & $2 \mathrm{~cm}$ \\
\hline Elongation before Yield & $<1 \mathrm{~mm}$ & $>2 \mathrm{~cm}$ & $>0.4 \mathrm{~cm}$ \\
\hline Yield Length \% & $<1.25 \%$ & $>100 \%$ & $>20 \%$ \\
\hline Yield Strength & $0.4 \mathrm{~N}$ & $0.4 \mathrm{~N}$ & $0.6 \mathrm{~N}$ \\
\hline${\text { Ongoing Lifetime in } \mathbf{9 0}^{\circ} \mathbf{C}}$ & $>20$ days & $>20$ days & $>20$ days \\
\hline
\end{tabular}

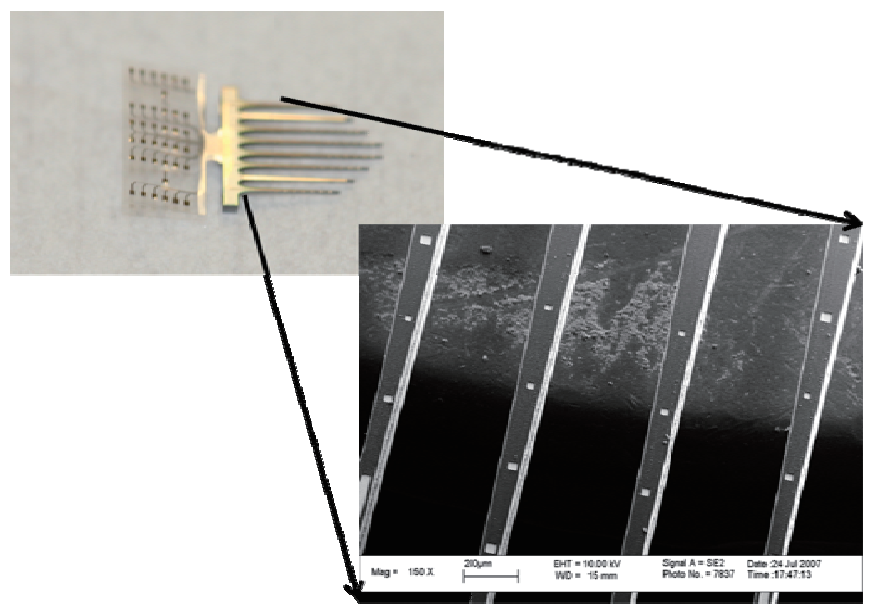

Figure 9: Silicon probe only structure for testing purpose. The parylene sheet extension provides metal pads for electrical connection to the parylene flexible cable. Conductive epoxy is used to make the electrical connection

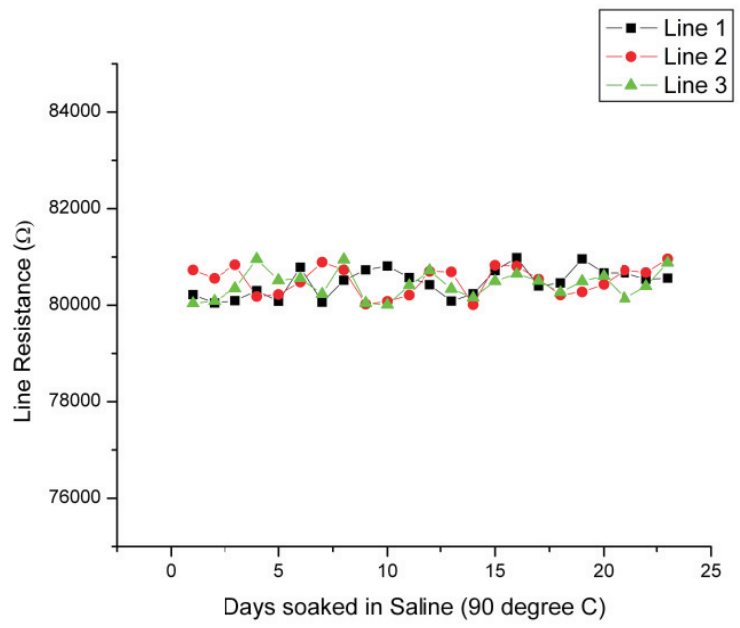

Figure 10: The metal line resistance in the cable measured as a function of days soaked in heated saline solution. The resistance is not affected by this test for at least 20 days.

\section{CONCLUSION}

We have developed and demonstrated the feasibility of a fully biocompatible, flexible and stretchable three dimensional coiled cable-interconnect that is able to be integrated with our current state of the art implantable prosthetic devices. The result of the mechanical pulling test is presented. High temperature accelerated life time testing in saline indicates the stability of the interconnect structure for more than 3 weeks in $90^{\circ} \mathrm{C}$ saline. From the testing results, this device shows promise as a versatile interconnect scheme to be incorporated into a future chronic cortical prosthetic system. Future work is underway to optimize the coating of the biocompatible silicone and the geometry of the cable.

\section{ACKOWLEDGEMENTS}

The authors would also like to thank Mr. Trevor Roper, Christine Garske and other members of the Caltech Micromachining Laboratory at Caltech for their generous advice and valuable assistance.

\section{REFERENCES}

[1] S. Musallam, B. D. Corneil, B. Greger, H. Scherberger, and R. A. Andersen, "Cognitive Control Signals for Neural Prosthetics," Science 305 (5681) pp. 258 - 262, 2004.

[2] R. Huang, C. Pang, Y.C. Tai, K. Emken, C. Ustun, R. Andersen, J. Burdick, "Integrated Parylene-Cabled Silicon Probes for Neural Prosthetics," in proc. MEMS 2008, pp. 240-243

[3] W. Li, D. Rodger, Y.C. Tai, "Implantable RF-Coiled Chip Packaging," in proc. MEMS 2008, pp. 108-111

[4] J. F. Hetke, K. Najafi, and K. D. Wise, "Flexible Silicon Interconnects for Microelectromechanical Systems," in proc. Transducers 1991, pp 764-767

[5] J. Subbaroyan and D. R. Kipke, "The role of flexible polymer interconnects in chronic tissue response induced by intracortical microelectrodes - a modeling and an in vivo study", in proc. EMBS 2006, pp 3588-3591 